\title{
Perfil comunicativo de crianças com Alterações Específicas no Desenvolvimento da Linguagem: caracterização longitudinal das habilidades pragmáticas
}

\section{Communicative profile of children with Language Impairment: longitudinal characterization of pragmatic abilities}

\author{
Debora Maria Befi-Lopes ${ }^{1}$, Marina Leite Puglisi ${ }^{2}$, Amalia Rodrigues ${ }^{3}$, Elisabete Giusti ${ }^{4}$, Juliana Perina Gândara ${ }^{5}$, \\ Karina de Araújo
}

\begin{abstract}
RESUMO
Objetivo: Analisar o desempenho longitudinal de crianças com Alterações Específicas no Desenvolvimento da Linguagem (AEDL) em uma prova de Pragmática a fim de observar o desenvolvimento da competência comunicativa ao longo do processo terapêutico. Métodos: Participaram desta pesquisa 56 crianças de ambos os sexos, com idades entre 1;10 (anos;meses) a 5;11 na primeira avaliação. Todos os sujeitos foram diagnosticados com AEDL e estavam/estiveram em terapia fonoaudiológica por pelo menos dois anos ou receberam alta antes deste período. As provas de Pragmática que faziam parte das avaliações anuais dos pacientes foram analisadas e pontuadas de 0 a 4 (pior ao melhor desempenho, respectivamente) com base em critérios de gravidade. Os resultados foram tratados estatisticamente (pd"0,05; ANOVA, Tukey-T, Pearson). Resultados: A idade das crianças com AEDL no momento do ingresso no serviço não foi uma variável relevante para determinar seus desempenhos na primeira avaliação de Pragmática, exceto para a idade de um ano $(\mathrm{p}=0,02)$, nem para predizer o tempo de terapia necessário para que eles normalizassem suas habilidades pragmáticas $(\mathrm{p}=0,72)$. As crianças que atingiram os critérios de normalidade para a prova $(64,3 \%)$ apresentaram um desempenho inicial melhor do que os sujeitos que não normalizaram seus desempenhos até o momento da coleta $(\mathrm{p}=0,01)$. Finalmente, enquanto o meio comunicativo foi o parâmetro que mais melhorou após terapia fonoaudiológica, o número de atos por minuto foi a variável cujo prejuízo mostrou-se mais persistente. Conclusão: O desempenho inicial das crianças com AEDL foi a variável que melhor predisse a normalização das habilidades pragmáticas dos sujeitos (64,3\%), após terapia fonoaudiológica.
\end{abstract}

Descritores: Desenvolvimento da linguagem; Transtornos do desenvolvimento da linguagem/fisiopatologia; Transtornos do desenvolvimento da linguagem/terapia; Comportamento verbal; Comunicação; Testes de linguagem

Trabalho realizado no Laboratório de Investigação Fonoaudiológica em Desenvolvimento da Linguagem e suas Alterações do Curso de Fonoaudiologia da Faculdade de Medicina da Universidade de São Paulo USP - São Paulo (SP), Brasil. O presente estudo é parte de projeto de pesquisa apoiado pelo CNPq, sob o número 470539/04-6.

(1) Livre docente; Professora do Departamento de Fisioterapia, Fonoaudiologia e Terapia Ocupacional da Faculdade de Medicina da Universidade de São Paulo - USP - São Paulo (SP), Brasil.

(2) Pós-graduanda do Programa de Pós-graduação em Ciências da Reabilitação da Faculdade de Medicina da Universidade de São Paulo - USP - São Paulo (SP), Brasil.

(3) Doutora, Fonoaudióloga Assistente do Departamento de Fisioterapia, Fonoaudiologia e Terapia Ocupacional da Faculdade de Medicina da Universidade de São Paulo - USP - São Paulo (SP), Brasil.

(4) Doutora, Coordenadora da Equipe de Diagnóstico da Associação de Pais e Amigos dos Excepcionais de Limeira - APAE - Limeira (SP), Brasil.

(5) Pós-graduanda do Programa de Pós-graduação em Lingüística da Faculdade de Filosofia, Letras e Ciências Humanas da Universidade de São Paulo - USP - São Paulo (SP), Brasil.

(6) Doutora, Colaboradora do Laboratório de Investigação Fonoaudiológica em Desenvolvimento da Linguagem e suas Alterações do Curso de Fonoaudiologia da Faculdade de Medicina da Universidade de São Paulo USP - São Paulo (SP), Brasil.

Endereço para correspondência: Debora M. Befi-Lopes. R. Cipotânea, 51, Cidade Universitária, São Paulo-SP, CEP 05360-160.E-mail: dmblopes@ usp.br Recebido em: 15/7/2007; Aceito em: 30/11/2007

\section{INTRODUÇÃO}

Entendida como o ramo da lingüística que estuda a relação entre o significado social da linguagem (expresso pelo contexto interacional) e seu conteúdo semântico (manifestado pelo significado do ato comunicativo em si), a pragmática refere-se ao uso efetivo da linguagem e aos seus propósitos funcionais de comunicação. Para isso, apóia-se nos diferentes significados intrínsecos aos processos comunicativos, determinados pelas informações extra-lingüísticas (pistas contextuais e situacionais) e pelas mensagens lingüísticas (semântica explícita). As alterações pragmáticas de linguagem são manifestadas por dificuldades em interpretar corretamente as ações dos outros e/ou em expressar adequadamente seus desejos e intenções. Portanto, os transtornos pragmáticos costumam ser constituídos causal ou consequientemente por um prejuízo nos componentes expressivo e receptivo de linguagem ${ }^{(1)}$.

As crianças com Alterações Específicas no Desenvolvimento da Linguagem (AEDL), embora constituam um grupo extremamente heterogêneo, compartilham de algumas carac- 
terísticas que fazem parte, justamente, dos critérios diagnósticos de inclusão e exclusão para a patologia. Resumidamente, as AEDL são diagnosticadas na presença de comprometimento em duas ou mais áreas da linguagem, concomitante à ausência de qualquer déficit neurológico, psiquiátrico, físico/ sensorial ou intelectual ${ }^{(2-5)}$. No entanto, é importante fazer uma distinção entre as AEDL representadas por retardo ou por distúrbio de linguagem. O retardo é caracterizado por um atraso generalizado na aquisição ou expressão de todos os componentes da linguagem que, ao se desenvolverem, acompanham a mesma seqüência do desenvolvimento normal. O distúrbio é configurado por um desenvolvimento atípico e discrepante das habilidades de linguagem, ou seja, por uma assincronia na aquisição de seus componentes em virtude de déficits específicos em certos aspectos lingüísticos ${ }^{(6)}$. De acordo com alguns autores $^{(5)}$, o diagnóstico diferencial entre retardo ou distúrbio de linguagem deve ser baseado em aspectos evolutivos a fim de buscar caracterizar a gravidade da AEDL, o ritmo de evolução do sujeito em cada componente da linguagem e as alterações associadas (maiores dificuldades atencionais, estimulação ambiental orientada, comportamento da criança).

Com base nestas definições, e tendo em mente o importante prejuízo lingüístico característico das crianças com AEDL, torna-se de grande relevância analisar as habilidades pragmáticas deste grupo de crianças. Tal relevância justifica-se pela possibilidade de detectar se, a despeito da falha em expressar o exato conteúdo semântico por meio da linguagem verbal, estas crianças possuem capacidade e/ou habilidade de expressar suas idéias e desejos a partir da linguagem não verbal, fazendo-se compreender e engajando-se em situações interacionais. Além disso, esta análise permite observar o quanto as dificuldades lingüísticas acabam por influenciar, e assim, prejudicar, o desenvolvimento de habilidades interacionais que não constituem o déficit primário da patologia, como, por exemplo, a intenção e a iniciativa comunicativas.

Esta questão foi abordada, inicialmente, por pesquisadores $^{(7-8)}$ que identificaram um prejuízo das habilidades pragmáticas em crianças com Distúrbio Específico de Linguagem (DEL), enquanto subgrupo das AEDL, tanto para os subtipos puramente expressivos, quanto para os mistos (expressivos e receptivos).

Mais recentemente, os estudiosos têm consumido esforços para identificar as relações de causalidade entre as alterações estruturais e funcionais presentes em patologias nas quais existe um comprometimento de linguagem. A intenção comum entre esses pesquisadores é a de caracterizar o desempenho pragmático de crianças de diferentes patologias (especialmente o das crianças com DEL e com Desordens do Espectro Autístico - DEA) e assim promover informações que possam auxiliar no diagnóstico diferencial entre os grupos. No entanto, esta tarefa não tem sido fácil. Os estudiosos têm encontrado muitas semelhanças entre os déficits pragmáticos e linguiísticos apresentados por certos grupos de crianças com DEL e com DEA. Fica difícil, desta forma, entender se os déficits pragmáticos das crianças com DEL são decorrentes de suas dificuldades relacionadas às áreas formais da linguagem (fonologia, semântica, morfossintaxe), ou se constituem uma falha mais abrangente, presente em todo o continuum existente entre as crianças com DEL e com DEA.

Para dar um panorama geral de tais estudos, podemos citar alguns dos mais relevantes acerca do tema, realizados nos últimos sete anos. Algumas pesquisas tiveram como objetivo comparar o tipo e a qualidade das respostas de três diferentes grupos de crianças: as com Prejuízo Pragmático de Linguagem (PPL); as com DEL; e aquelas com desenvolvimento típico de linguagem. Os autores ${ }^{(9)}$ identificaram que as crianças com PPL apresentaram menos respostas às solicitações de seus pais do que as demais, e que estas respostas eram qualitativamente piores. Deve-se, no entanto, ter cuidado ao interpretar as dificuldades pragmáticas apresentadas pelas crianças com prejuízos comunicativos dado que, em muitos momentos, a sintomatologia de crianças com DEL e com DEA pode confundir-se ${ }^{(10)}$. Esta semelhança entre os grupos pode acontecer tanto em relação às habilidades pragmáticas quanto a algumas habilidades lingüísticas, se analisadas isoladamente. Em outro estudo ${ }^{(11)}$, estas mesmas autoras analisaram o desempenho das crianças com DEL, com PPL e com Autismo de Alto Funcionamento (AAF) em prova de compreensão literal e inferencial de histórias. Elas concluíram que, embora os diferentes grupos possuam algumas características peculiares, o desempenho de todos não difere pela performance nesta prova. Elas acreditam que a compreensão prejudicada, mesmo que em graus e com características diferentes em cada um dos grupos estudados, altera a formação de representações mentais estáveis e apropriadas sobre as histórias, levando a uma dificuldade, também, para recontá-las adequadamente.

Outras pesquisadoras ${ }^{(4)}$ buscaram encontrar as diferentes marcas clínicas referentes às habilidades lingüísticas de crianças diagnosticadas com DEL, com PPL e com DEA. As autoras concluíram que todos os grupos apresentaram um pior desempenho em todas as medidas psicolingüísticas testadas, embora tenha havido diferença com relação à performance de cada grupo de sujeitos. Outro grupo de estudiosos $^{(12)}$ identificou que $21 \%$ das crianças diagnosticadas com DEL apresentavam dificuldades pragmáticas mesmo quando não demonstravam falhas nas habilidades lingüísticas formais ou no comportamento não verbal. Complementando estes achados, há evidências que sugerem a existência de influência genética tanto para as alterações estruturais quanto funcionais de linguagem ${ }^{(13)}$.

As pesquisas acima mencionadas, embora enfoquem especificamente os casos de DEL, amplamente estudados em decorrência da complexidade do quadro, indicam que ainda é difícil realizar o diagnóstico diferencial com base apenas nas habilidades lingüísticas ou pragmáticas, consideradas isoladamente. Fica claro, no entanto, que as crianças com AEDL, ao menos aquelas com diagnóstico de DEL, apresentam dificuldades pragmáticas de linguagem. Tais dificuldades podem ser caracterizadas, dentre outras, por: maior número de respostas inadequadas do que as crianças em desenvolvimento típico de linguagem, sem melhora significante com o aumento da idade ${ }^{(14)}$; dificuldade de iniciar atos comunicativos e menor sucesso ao fazê-lo do que seus pares em desenvolvimento normal, necessitando inclusive de mais 
tempo para engajarem em uma situação comunicativa ${ }^{(15)}$; e persistência, até a adolescência, da baixa competência social para realizar amizades e do alto risco de vitimização apresentado por esses sujeitos ${ }^{(16)}$.

Todos esses dados indicam que o déficit pragmático, além de existente, é bastante salientado nas crianças com AEDL, mas a dificuldade em rastrear as marcas clínicas comuns à patologia e em estabelecer relações de causalidade, ao menos em idades precoces (antes dos cinco anos, quando ainda é complexa a diferenciação entre Retardos de Linguagem e Distúrbios Específicos de Linguagem), demonstram a necessidade de um maior número de estudos sobre o tema.

Desta forma, o objetivo desta pesquisa foi analisar comparativamente o desempenho evolutivo das crianças com AEDL na avaliação da pragmática, a fim de mensurar o grau de competência comunicativa que elas são capazes de adquirir ao longo do processo terapêutico, seja pelas habilidades verbais e/ou não verbais. Por se tratar de um estudo longitudinal, em particular, esta pesquisa viabiliza a determinação de quais aspectos pragmáticos são passíveis de melhora ao longo do processo terapêutico, e quais acabam por constituir, de certa forma, algumas das marcas clínicas da patologia, em específico nos casos de DEL. O presente estudo fez parte de um projeto de pesquisa mais abrangente (Perfil Comunicativo de Crianças com Alterações de Desenvolvimento da Linguagem - Caracterização Longitudinal), que tinha como objetivo principal caracterizar longitudinalmente o padrão de comunicação apresentado por crianças com diagnóstico de AEDL, a partir da verificação da evolução de seus desempenhos em provas específicas de Vocabulário, Fonologia, Pragmática, Fluência, Extensão Média do Enunciado e Maturidade Simbólica. A partir desse objetivo, foram formuladas hipóteses individuais para cada habilidade testada. Este estudo pretende descrever os resultados obtidos em relação à avaliação da Pragmática, e testou as seguintes hipóteses:

- H1: As crianças que realizaram a primeira avaliação com idade mais avançada apresentariam pior desempenho em prova de Pragmática.

- H2: As crianças que realizaram a primeira avaliação com idade mais avançada necessitariam de maior tempo de terapia fonoaudiológica para normalizar (atingir o desempenho esperado para a faixa etária) a habilidade de pragmática.

- H3: As crianças que chegaram a normalizar a habilidade de pragmática apresentariam melhor desempenho na avaliação inicial quando comparadas às que não normalizaram.

\section{MÉTODOS}

\section{Aspectos Éticos}

Esta pesquisa foi subtida à avaliação pela Comissão de Ética para Análise de Projetos de Pesquisa do HCFMUSP (CAPPesq) e aprovada segundo o parecer de $n^{\circ}$ 552/06. Todos os responsáveis pelos pacientes assinaram termo de consentimento livre e esclarecido para participação na pesquisa.

\section{Sujeitos}

Participaram desse estudo os pacientes que estiveram ou ainda encontram-se em terapia fonoaudiológica no Laboratório de Investigação Fonoaudiológica em Desenvolvimento da Linguagem e Suas Alterações (LIF-ADL) do Departamento de Fisioterapia, Fonoaudiologia e Terapia Ocupacional da Faculdade de Medicina da Universidade de São Paulo (FMUSP). Todos os sujeitos tiveram diagnóstico de AEDL, realizado com base em critérios de inclusão e exclusão internacionalmente descritos (alterações de linguagem na ausência de outros comprometimentos clínicos, como perdas auditivas, lesões neurológicas marcadas, rebaixamento intelectual, privação neurossensorial ou comprometimentos emocionais de ordem abrangente) $)^{(17-19)}$.

A seleção dos sujeitos desta pesquisa levou em consideração os seguintes critérios de inclusão:

- primeira avaliação realizada entre início de 2000 e início de 2004;

- pelo menos duas avaliações realizadas (foram consideradas as avaliações com intervalo de um ano entre si, a partir da data da avaliação inicial);

- exclusão dos sujeitos que não se encaixavam em um dos seguimentos possíveis: alta, encaminhamento para atendimento em decorrência de alterações fonológicas leves ou alterações de fluência (nesses dois primeiros casos, independente do número de avaliações realizadas), abandono do processo terapêutico (considerados apenas os sujeitos que tivessem pelo menos duas avaliações) ou ainda em terapia.

De acordo com esses critérios, foram recrutadas 56 crianças com idades entre 1;9 (anos;meses) e 5;2 na primeira avaliação, momento em que foi realizado o diagnóstico.

\section{Material}

Foram analisadas as provas de Pragmática ${ }^{(20-21)}$ realizadas pelos sujeitos em suas avaliações anuais. Esta prova consiste na identificação e interpretação dos atos comunicativos apresentados pelo paciente em uma situação de interação livre, na qual seja possível à criança desenvolver uma brincadeira prazerosa e interagir com a avaliadora. A prova de Pragmática visa registrar os dados que reflitam a efetividade e competência comunicativas da criança, e caracterizá-los com base em três critérios específicos:

- Número de atos por minuto - reflete a iniciativa comunicativa, pois é calculado pela quantidade de atos iniciados pela criança durante a interação com a terapeuta.

- Meio comunicativo predominante - reflete a competência verbal, pois é analisado pela quantidade de uso dos meios verbal, vocal e/ou gestual, nos atos apresentados pela criança.

- Função comunicativa predominante - reflete a competência funcional, pois é caracterizada pelo tipo de função social que os atos realizados pela criança representam.

Todas as sessões de avaliação foram gravadas durante 15 minutos (em fita VHS e em fita cassete) para posterior transcrição e análise, ambas realizadas pelas terapeutas de cada 
paciente, na época da avaliação.

Os dados usados para o presente estudo, coletados segundo os critérios acima descritos, foram retirados dos prontuários de cada criança.

\section{Procedimentos}

Após o levantamento dos prontuários, todos os protocolos de pragmática de cada sujeito foram tabulados de acordo com os critérios elaborados especialmente para esta pesquisa, que serão descritos a seguir.

Os aspectos considerados para a pontuação em cada prova de cada paciente foram os seguintes: número de atos por minuto, meio comunicativo e funções comunicativas. Para cada um destes itens, atribuiu-se 1 ponto para os aspectos adequados e 0 para os inadequados. Assim, inicialmente, cada criança poderia obter uma pontuação que variava entre 0 e 3 pontos em cada prova, conforme especificado a seguir:

\section{Número de atos por minuto}

Para analisar o número de atos por minuto, foram usados dois diferentes valores de referência, de acordo com a idade da criança (Quadro 1).

Quadro 1. Valores de referência para o número total de atos por minuto na prova de Pragmática

\begin{tabular}{|c|c|c|}
\hline Referência & Faixa etária & Número de atos/minuto \\
\hline Fernandes, 2004(21) & $1 ; 0$ & 3,00 \\
\hline Rodrigues, $2002^{(22)}$ & $2 ; 0$ & 5,52 \\
\hline Rodrigues, $2002^{(22)}$ & $3 ; 0$ & 5,70 \\
\hline Rodrigues, 2002(22) & $4 ; 0$ & 7,16 \\
\hline Fernandes, 2004(21) & $5 ; 0$ & 8,00 \\
\hline Fernandes, 2004(21) & $7 ; 6$ & 9,00 \\
\hline Fernandes, 2004(21) & Adulto & 10,00 \\
\hline
\end{tabular}

De acordo com esses valores de referência, foi atribuída a seguinte pontuação ao número total de atos por minuto em cada prova:

- 0 para as provas cujos atos estavam abaixo do esperado para a idade

- 1 para as provas cujos atos estavam dentro do esperado para a idade

\section{Funções comunicativas}

Com base em critérios previamente definidos ${ }^{(22)}$, as funções comunicativas foram classificadas em:

- mais interacionais (Pedido de Objeto; Reconhecimento do Outro; Exclamação; Protesto; Narrativa; Pedido de Solicitação; Comentário; Pedido de Ação; Exibição; Jogo Compartilhado; Pedido de Informação; e Pedido de Consentimento).

- menos interacionais (Expressão de Protesto; Performativa; Não Focalizada; Reativa; Nomeação; Exploratória; Autorregulatória; e Jogo).

Tendo em vista essa classificação, as funções referentes aos atos apresentados pelas crianças foram pontuadas da seguinte forma:

- 0 para as provas cuja função predominante era menos interacional

- 1 para as provas cuja função predominante era mais interacional ou se havia o mesmo número de funções mais e menos interacionais

\section{Meio comunicativo}

Levando-se em consideração que as crianças mais jovens da pesquisa já tinham idade para conseguir produzir corretamente $75 \%$ dos fonemas da língua, o meio comunicativo foi pontuado da seguinte forma:

- 0 para as provas cujo meio predominante era vocal (VO) ou gestual $(\mathrm{G})$

- 1 para as provas cujo meio predominante era verbal (VE).

Em alguns casos específicos, no entanto, as provas realizadas pelas crianças não puderam ser pontuadas, pois se enquadravam no critério "Não Analisável" (NA), caracterizado por uma falha no procedimento de aplicação da prova e/ ou da transcrição dos dados.

Para a pontuação final, os três quesitos já mencionados foram associados a um outro critério: a não realização da prova por inadequações de comportamento da criança, que impediram a aplicação ou a análise apropriada da prova. Nos casos em que isso ocorreu, a pontuação atribuída à criança naquela prova foi 0 . As crianças que realizaram a prova (indepedentemente da qualidade) somaram 1 ponto à pontuação final. O Quadro 2 demonstra os critérios adotados para a pontuação na prova de Pragmática.

\section{Análise dos dados}

Para responder as hipóteses iniciais, foram realizadas diferentes análises estatísticas, a fim de promover o tratamento mais adequado dos dados. Os testes estatísticos utilizados foram ANOVA, teste de Tukey e correlação de Pearson. O pvalor de corte adotado foi de 0,05 .

Quadro 2. Esquema de pontuação para a Prova de Pragmática

\begin{tabular}{|lccc|}
\hline Desempenho na prova & Pontuação para realização da prova & Pontuação nos 3 quesitos & Pontuação final \\
\hline Não Realizada (NR) & 0 & - & 0 \\
Nenhum dos quesitos adequado & 1 & 1 & 1 \\
1 dos 3 quesitos adequado & 1 & 2 & 3 \\
2 dos 3 quesitos adequados & 1 & 3 & 4 \\
Todos os quesitos adequados & 1 & 1 & \\
\hline
\end{tabular}




\section{RESULTADOS}

Os resultados serão descritos com base nas hipóteses levantadas nesse estudo.

\section{Relações entre idade inicial e desempenho dos sujeitos}

$\mathrm{Na}$ tentativa de indentificar uma possível medida de prognóstico terapêutico, foram feitas duas análises que levaram em conta a idade de cada sujeito no momento em que eles ingressaram no serviço.

Para testar a $\mathrm{H} 1$, verificamos, conforme demonstrado na Tabela 1, a relação existente entre a idade de cada sujeito na primeira avaliação e seu desempenho nesta mesma prova. Utilizamos, para tal, o teste ANOVA.

Tabela 1. Análise da relação existente entre a idade dos sujeitos na primeira avaliação de pragmática e as médias de seus desempenhos nesta prova

\begin{tabular}{lccccc}
\hline Fonte & $\mathrm{GL}$ & $\mathrm{SQ}$ & $\mathrm{MQ}$ & Teste F & P-valor \\
\hline Idade & 4 & 9,75 & 2,44 & 3,34 & 0,02 \\
Erro & 47 & 34,31 & 0,73 & & \\
\hline Total & 51 & 44,06 & & &
\end{tabular}

Legenda: GL - Graus de liberdade; SQ - Soma de Quadrados; MQ - Média de Quadrados (SQ/GL)

Os resultados indicaram que a idade é uma variável relevante para o desempenho dos sujeitos na prova de pragmática. No entanto, ao realizar o teste de Tukey, cujo objetivo era localizar tal diferença entre as diferentes faixas etárias (Tabela 2), verificamos que apenas o desempenho das crianças de um ano diferiu do das demais.

Tabela 2. Comparação das médias de desempenho na prova de pragmática, entre cada faixa etária

\begin{tabular}{lccccc}
\hline Idade & 1 ano & 2 anos & 3 anos & 4 anos & 5 anos \\
\hline Média & 0,0 & 2,23 & 2,25 & 2,25 & 2,0 \\
\hline $\begin{array}{l}\text { Diferença } \\
\text { Significante }\end{array}$ & $\mathrm{C} / 2,3,4,5 \mathrm{a}$ & $\mathrm{C} / 1 \mathrm{a}$ & $\mathrm{C} / 1 \mathrm{a}$ & $\mathrm{C} / 1 \mathrm{a}$ & $\mathrm{C} / 1 \mathrm{a}$ \\
\hline
\end{tabular}

Desta forma, ao assumir que a relevância da idade para o desempenho nas provas de pragmática existiu apenas para as crianças de um ano, entendemos que esta variável não pode ser considerada, de uma forma geral, como relevante para a performance dos sujeitos nesta prova.

Em seguida, para testar a $\mathrm{H} 2$, avaliamos se a mesma variável (idade dos sujeitos na primeira avaliação) influenciou no tempo de terapia fonoaudiológica necessário para normalizar o desempenho na prova de pragmática. Para tal, tivemos que selecionar apenas os indivíduos que conseguiram, em algum momento, contemplar os critérios de normalidade. Desta forma, dos 56 sujeitos, apenas 36 (64,3\%) foram recrutados para esta análise (sujeitos que tiveram alta, receberam EE ou alcançaram a normalidade nesta habilidade, mas permaneceram em terapia fonoudiológica em virtude de manterem outros déficits de linguagem).
Inicialmente, foi necessário calcular previamente o tempo que os sujeitos demoraram para normalizar o desempenho. Considerando que cada sujeito foi analisado longitudinalmente, com intervalo de um ano entre cada avaliação, calculamos que a média total de tempo de terapia (em anos) necessária para que ocorresse a normalização do desempenho foi de 2,14 anos, para os sujeitos desta análise. Fizemos, posteriormente, a mesma análise para cada faixa etária e observamos que o tempo de terapia foi muito semelhante para todos os sujeitos, independentemente de suas idades (Tabela 3).

Tabela 3. Comparação das médias de tempo de terapia necessário para a normalização do desempenho, entre cada faixa etária

\begin{tabular}{lccccc}
\hline Idade & 1 ano & 2 anos & 3 anos & 4 anos & 5 anos \\
\hline Média & 2,5 & 1,9 & 2,1 & 2,5 & 2,2 \\
\hline
\end{tabular}

Para verificar a significância estatística desta análise anterior (variação do tempo de terapia por faixa etária), aplicamos a ANOVA e concluímos que, de fato, a idade não foi uma variável relevante para determinar o tempo que os sujeitos demoraram para atingir a normalidade (Tabela 4).

Tabela 4. Análise da relação existente entre a idade dos sujeitos na primeira avaliação de pragmática e o tempo de terapia necessário para normalizar seus desempenhos nesta prova

\begin{tabular}{lccccc}
\hline Fonte & $\mathrm{GL}$ & $\mathrm{SQ}$ & $\mathrm{MQ}$ & Teste $\mathrm{F}$ & $\mathrm{P}$-valor \\
\hline Idade & 4 & 1,68 & 0,420 & 0,53 & 0,72 \\
Erro & 31 & 24,63 & 0,794 & & \\
\hline Total & 35 & 26,42 & & &
\end{tabular}

Legenda: GL - Graus de liberdade; SQ - Soma de Quadrados; MQ - Média de Quadrados (SQ/GL)

As análises até agora descritas demonstraram que a idade dos sujeitos na primeira avaliação de pragmática não foi uma variável relevante para seus desempenhos na prova (exceto para as crianças de um ano) nem para o tempo que levaram para superar seus déficits pragmáticos. Assim, a idade inicial dos sujeitos, no momento do ingresso em terapia, não demonstrou ser uma boa medida prognóstica para as habilidades pragmáticas.

\section{Relações entre o desempenho na primeira avaliação e o tempo de terapia}

Nesta etapa, testamos se o desempenho dos sujeitos na primeira avaliação de pragmática estava relacionado à possibilidade de eles atingirem os critérios de normalidade nesta prova, ao longo do processo terapêutico. Assim, quisemos verificar se o desempenho inicial dos sujeitos teria algum valor preditivo e, assim, testar a $\mathrm{H} 3$.

Para avaliar este item, foi necessário comparar o desempenho inicial dos pacientes que conseguiram normalizar o desempenho ao longo do processo terapêutico (a mesma amostra citada para a análise da $\mathrm{H} 2$ ), com o desempenho dos pacientes que nunca normalizaram (sujeitos que não atingi- 
ram os critérios de normalidade e permaneceram em terapia fonoudiológica), conforme pode ser observado na Tabela 5 . Os pacientes que abandonaram a terapia e os que já tinham desempenho adequado na primeira avaliação foram desconsiderados nesta análise.

Tabela 5. Comparação entre o desempenho inicial dos sujeitos que conseguiram normalizar e dos que não conseguiram, ao longo do processo terapêutico

\begin{tabular}{lccc}
\hline Normalizou & Não & Sim & P-valor \\
\hline Média & 1,63 & 2,33 & 0,01 \\
\hline
\end{tabular}

Observamos que os sujeitos que, em algum momento, atingiram os critérios de normalidade na prova de Pragmática, apresentaram um desempenho na avaliação inicial significantemente melhor do que aqueles que não conseguiram normalizar. Estes aspectos podem ser exemplificados como no Quadro 3.

\section{Correlações entre diferentes parâmetros na prova de pragmática}

Finalmente, foi aplicada a Correlação de Pearson aos dados julgados relevantes na análise da pragmática, a fim de verificar se existia alguma relação de dependência entre as variáveis (Tabela 6). Os itens correlacionados foram:

- Idade na primeira avaliação da pragmática (Idade)

- Desempenho na primeira avaliação da pragmática (D Inicial)

Tabela 6. Correlação entre as diferentes variáveis na prova de Pragmática

\begin{tabular}{lllll}
\hline p-valores & Idade & D Inicial & TTerapia & Normalizar \\
\hline Idade & - & & & \\
D Inicial & 0,28 & - & & \\
TTerapia & 0,67 & 0,89 & - & \\
\hline Correlação & Idade & D Inicial & TTerapia & Normalizar \\
\hline Idade & 1,00 & & & \\
D Inicial & 0,15 & 1,00 & & \\
T Terapia & 0,07 & $-0,02$ & 1,00 & \\
Normalizar & $-0,09$ & 0,33 & 0,00 & 1,00 \\
\hline
\end{tabular}

- Tempo de terapia necessário para atingir os critérios de normalidade ( $\mathrm{T}$ Terapia)

- Possibilidade do sujeito normalizar o desempenho da prova, em algum momento (Normalizar)

Os resultados indicam que houve correlação positiva significante apenas entre o desempenho na primeira avaliação da pragmática e a possibilidade do sujeito normalizar o desempenho nesta prova. Assim, quanto melhor o desempenho inicial dos sujeitos, maior é a chance de ele um dia normalizar seu desempenho nesta prova e, assim, compensar os déficits pragmáticos.

\section{DISCUSSÃO}

De acordo com as hipóteses levantadas no início do estudo, foi possível chegar a importantes achados, que serão discutidas a seguir.

H1: As crianças que realizaram a primeira avaliação com idade mais avançada apresentariam pior desempenho em prova de Pragmática - hipótese rejeitada.

Apenas o desempenho inicial das crianças de um ano de idade apresentou diferença estatisticamente significante com relação ao desempenho das crianças dos demais grupos. Dado que o número de sujeitos que compunham o grupo de crianças de um ano foi bastante reduzido (apenas duas crianças), é possível considerar que o desempenho dos sujeitos na prova de Pragmática independeu da idade com que eles ingressaram no serviço, em busca de atendimento fonoaudiológico. Em outras palavras, poderíamos dizer que o déficit pragmático, existente nas crianças com AEDL, não diferiu em termos de gravidade de acordo com o tempo que estes sujeitos demoraram para iniciar o tratamento. Vale ressaltar que as idades dos sujeitos no momento do início da terapia fonoudiológica variou de 1;9 anos a 5;2. Embora alguns estudos tenham tentado identificar, cada vez mais precocemente, as marcas clínicas iniciais características dos Retardos de Linguagem (RL) e dos DEL ${ }^{(23-24)}$, a ausência de consenso na literatura sobre as caraterísticas preditoras da patologia faz com que somente aos cinco anos seja possível diferenciar, com mais segurança, estes subgrupos das AEDL. Assim, a idade inicial dos sujeitos de nosso estudo não tendeu, por si só, a separar as crianças que apresentavam RL daquelas com DEL, representando uma possível explicação do porque a

Quadro 3. Perfil longitudinal do desempenho pragmático das crianças com AEDL

\begin{tabular}{|c|c|c|c|}
\hline \multirow[t]{2}{*}{ Avaliação } & \multicolumn{3}{|c|}{ Desempenho na prova de Pragmática } \\
\hline & $\begin{array}{l}\text { Média - atos por minuto } \\
\quad(\text { variação 0-1) }\end{array}$ & $\begin{array}{c}\text { Média - meio comunicativo } \\
\text { (variação 0-1) }\end{array}$ & $\begin{array}{c}\text { Média - funções comunicativas } \\
\text { (variação 0-1) }\end{array}$ \\
\hline $1^{\text {a }}$ avaliação & 0,10 & 0,20 & 0,83 \\
\hline $2^{\mathrm{a}}$ avaliação & 0,22 & 0,52 & 0,96 \\
\hline $3^{\mathrm{a}}$ avaliação & 0,27 & 0,76 & 0,93 \\
\hline $4^{\mathrm{a}}$ avaliação & 0,05 & 0,90 & 0,95 \\
\hline $5^{\mathrm{a}}$ avaliação & 0,00 & 1,00 & 1,00 \\
\hline $6^{a}$ avaliação & 0,25 & 1,00 & 1,00 \\
\hline
\end{tabular}


idade na primeira avaliação não foi relevante para prever o desempenho dos sujeitos. É possível questionar, no entanto, se a inclusão de sujeitos com mais de cinco anos à época do ingresso ao serviço poderia levar a outros resultados.

H2: As crianças que realizaram a primeira avaliação com idade mais avançada necessitariam de maior tempo de terapia fonoaudiológica para normalizar (atingir o desempenho esperado para a faixa etária) a habilidade de pragmática - hipótese rejeitada.

A idade dos sujeitos na avaliação inicial não foi, da mesma forma, relevante para determinar o tempo de terapia fonoaudiológica necessário para normalizar o desempenho na avaliação da Pragmática. Esse dado reforça o resultado anterior, demonstrando que a gravidade do déficit pragmático não foi determinada pela idade com que os sujeitos iniciaram a terapia fonoaudiológica, dado que os indivíduos mais velhos necessitaram de um número de terapia semelhante ao dos mais novos, até normalizar o desempenho.

É importante ressaltar, neste momento, que os sujeitos desta pesquisa necessitaram, em média, de 64 sessões terapêuticas (número aproximado de sessões referentes a 2,14 anos, descontando-se os períodos de férias e feriados) para normalizar seus desempenhos na prova de Pragmática, um tempo bastante longo. Esse resultado é compreensível na medida em que esta prova reflete o perfil funcional de comunicação das crianças, e, portanto, pode ser considerada uma manifestação secundária de déficits mais específicos, como o fonológico, o semântico e o morfossintático, que compõem a base do domínio lingüístico. De fato, estudio$\operatorname{sos}^{(15)}$ observaram que as medidas de linguagem expressiva, apresentadas pelas crianças com DEL, foram positivamente correlacionadas à porcentagem de enunciados produzidos pelas mesmas para inciar uma situação comunicativa com seus colegas. Estes enunciados incluem os três parâmetros avaliados na prova de Pragmática deste estudo, pois contemplam a iniciativa comunicativa (número atos), o uso do meio verbal e a utilização de funções comunicativas mais interacionais. Desta forma, é natural que a normalização do desempenho de crianças com AELD na prova de Pragmática, por ser em grande parte reflexo da melhora em outras habilidades lingüísticas, demore para ser alcançada.

H3: As crianças que chegaram a normalizar a habilidade de pragmática apresentariam melhor desempenho na avaliação inicial quando comparadas às que não normalizaram - hipótese confirmada.

As crianças que conseguiram atingir os critérios de normalidade, ao longo do processo terapêutico, obtiveram, de fato, uma pontuação na primeira avaliação da Pragmática melhor do que aquelas que não foram capazes de superar o déficit. Entretanto, é muito importante sinalizar um fato relevante: embora a diferença entre os grupos tenha sido considerada estatisticamente significante, ambos apresentaram um desempenho ruim na avaliação inicial, o que pode ser demonstrado pela média da pontuação $(2,33$ para as crianças que normalizaram e 1,63 para os sujeitos que não normalizaram até o momento da coleta dos dados) e está de acordo com os dados encontrados na literatura ${ }^{(14-16)}$. De acordo com a pontuação estabelecida e descrita anteriormente, devemos lembrar que a pontuação 1 foi atribuída àqueles indivíduos que se encontravam abaixo em todos os quesitos analisados, enquanto a pontuação 2 referia-se àqueles indivíduos que tivessem apenas $u m$ dos critérios adequados. Ao resgatar as planilhas de dados dos pacientes, pudemos observar que tal critério adequado era, quase em sua totalidade (exceto para um sujeito), o tipo de função comunicativa mais utilizada. Assim, podemos deduzir que as crianças que apresentaram, na avaliação inicial, ao menos uma quantidade predominante de funções comunicativas mais interacionais, tiveram maior chance de um dia alcançarem os padrões de normalidade na prova de Pragmática. Reforçando essas conclusões, podemos enfatizar que houve correlação positiva significante entre o desempenho dos sujeitos na primeira avaliação da Pragmática e a possibilidade deles normalizarem o desempenho nesta prova. Estes dados, além de interessantes, parecem bastante relevantes em termos de prognóstico terapêutico.

De todos os 56 sujeitos deste estudo, a grande maioria $(64,3 \%)$ normalizou o desempenho na prova de pragmática, enquanto uma parcela significativa $(19,6 \%)$ não atingiu os critérios de normalidade até o momento da coleta (o restante do grupo - 16,1\% - já era normal na avaliação inicial ou abandonou o serviço antes de normalização do desempenho). Este dado corrobora os achados de outro estudo ${ }^{(9)}$, no qual se concluiu que existe um grupo de crianças com prejuízos de linguagem mais amplos, que se estendem além das alterações lingüísticas (fonológicas, lexicais, morfossintáticas) para dificuldades em responder e expressar suas intenções comunicativas. $\mathrm{O}$ fato novo, concluído no presente estudo, referese à possibilidade de sinalizar, ainda que grosseiramente, quais crianças apresentariam maior risco para desenvolverem tais alterações mais severas e abrangentes, já na primeira avaliação de pragmática.

Se fizermos uma análise mais geral dos dados, a fim de observar as características do perfil longitudinal do desempenho dos sujeitos na prova de Pragmática, podemos verificar uma dinâmica interessante e pertinente. Conforme descrito anteriormente, os parâmetros de pontuação do desempenho das crianças na prova de Pragmática levaram em conta, igualmente, o número de atos por minuto, o meio comunicativo predominante e as funções comunicativas mais utilizadas. O perfil pragmático das crianças que ingressaram no serviço ( $1^{\text {a }}$ avaliação - Quadro 3$)$, demonstrado pela média do desempenho em cada aspecto analisado, evidencia que o déficit pragmático inicial apresentado pelas crianças com AEDL foi caracterizado, principalmente, pelo baixo número de atos por minuto e pela predominância dos meios comunicativos vocal e gestual. O tipo de função comunicativa mais utilizada parece não representar uma área de grande dificuldade, mesmo no início do processo terapêutico, demonstrando que a alteração pragmática da maioria das crianças não está relacionada à pertinência social da interação com o outro, mas principalmente à iniciativa comunicativa e à forma utilizada para tal, que dependem, especialmente, de habilidades lingüísticas. Esse dado, inclusive, é reforçado pela ampla literatura que demonstra que as crianças com 
AEDL apresentam menor eficiência comunicativa e habilidades conversacionais mais pobres em comparação com seus pares em desenvolvimento normal ${ }^{(12-16)}$.

Se observamos, ao contrário, o desenvolvimento do meio comunicativo, podemos percerber que este é o parâmetro cuja melhora fica mais evidente ao longo do processo terapêutico (Quadro 3). O baixo desempenho dos sujeitos com relaçao ao meio comunicativo, na primeira avaliação, pode ser entendido como decorrente da ausência de oralidade ou da presença de alterações fonológicas importantes e, portanto, reflexo da incompetência verbal dessas crianças. Assim, é de se esperar que o investimento terapêutico na melhora das habilidades semânticas e fonológicas, muito frequiente na prática clínica, promova um aumento do uso do meio verbal em situações de comunicação. Essa conclusão é pertinente à definição da própria patologia, que refere os déficits lingüísticos como suas manifestações primárias ${ }^{(25-32)}$.

Por fim, podemos entender que a baixa iniciativa comunicativa caracterizada pela baixa média de atos por minuto em todas as avaliações realizadas define, possivelmente, uma das marcas clínicas da patologia. Podemos notar que existe um aumento da média de atos apresentados pelos sujeitos, até a terceira avaliação (Quadro 3). Essa melhora, certamente, aconteceu para os sujeitos que conseguiram normalizar o desempenho nesta prova (tiveram alta; foram encaminhados para um serviço de atenção primária; ou alcançaram a normalidade nesta habilidade, mas permaneceram em terapia fonoudiológica em virtude de manterem outros déficits de linguagem). Eram, de qualquer forma, sujeitos que apresentavam quadros mais leves de alterações de linguagem. A partir da quarta avaliação, podemos notar uma importante queda da média de desempenho nesta habilidade, decorrente, possivelmente, da maior concentração de sujeitos com quadros mais graves de alterações de linguagem, enfatizada inclusive pela longa duração do processo terapêutico. Assim, podemos supor que a baixa iniciativa comunicativa constitui-se, de certa forma, como uma das marcas clínicas da patologia, fato que é embasado na literatura referente ao tema ${ }^{(9,13,15)}$.

\section{CONCLUSÃO}

Os resultados deste estudo indicaram que a idade das crianças com AEDL na primeira avaliação de Pragmática, realizada no momento do início do processo terapêutico, não foi uma variável relevante para o desempenho dos sujeitos nessa mesma prova, nem para o tempo de terapia que eles precisaram para normalizar as habilidades pragmáticas.

Além disso, as crianças que, ao longo do processo terapêutico, atingiram os critérios de normalidade para a prova, apresentaram um desempenho inicial melhor (caracterizado, principalmente, pelo uso de funções comunicativas adequadas) do que os sujeitos que não chegaram a normalizar seus desempenhos até o momento da coleta.

Finalmente, o perfil pragmático longitudinal das crianças com AEDL desta pesquisa demonstrou que o parâmetro mais passível de melhora, após terapia fonoaudiológica, foi o meio comunicativo, já que ao melhorar as habilidades semânticas e fonológicas, os pacientes tornam-se mais aptos a utilizar o meio verbal predominantemente em suas interações. O número de atos por minuto, ao contrário, demonstrou ser o parâmetro mais prejudicado nesta patologia, despontando, inclusive, como uma de suas possíveis marcas clínicas.

\begin{abstract}
Purpose: To analyze the longitudinal performance of children with Language Impairments (LI) on a pragmatic test, in order to observe the development of communicative abilities through speech and language therapy (SLT). Methods: Fifty six children from both sexes and ages ranging from 1;10 (years;months) and 5;11 in the first evaluation participated of this study. All subjects were diagnosed with Language Impairments (LI) and were engaged in language therapy for at least two years or were discharged before this period. We analyzed the pragmatic tests collected from patients' annual evaluations, since the beginning of SLT. Children's performance were scored from 0 to 4 (the worst to the best performance, respectively) based on severity criteria. The data was statistically analyzed using ANOVA, Tukey-T and Pearson, with a significance level of pd'0.05. Results: Age was not a relevant variable to determine subject's performance at the first pragmatic assessment, except for one-year-old children ( $\mathrm{p}=0,02$ ), neither to predict the number of SLT sessions children needed to overcome the pragmatic deficit $(\mathrm{p}=0,72)$. Children who eventually achieved normal scores on the pragmatic test $(64,3 \%)$ showed a better initial performance on that test than children who did not reach expected levels through the period considered in the study $(\mathrm{p}=0,01)$. Finally, whereas communicative mean was the parameter that mostly improved with SLT, the number of acts per minute was the variable the mostly remained impaired for children with LI. Conclusion: Initial performance of children with LI was more predictive of pragmatic abilities overcome (which happened to $64.3 \%$ of the sample) than other variables.
\end{abstract}

Keywords: Language development; Language development disorders/physiopathology; Language development disorders/therapy; Verbal behavior; Communication; Language tests 


\section{REFERÊNCIAS}

1. Munoz-Yunta JA, Palau-Baduell M, Salvado-Salvado B, VallsSantasusana A, Perich-Alsina X, Del Rio Grande D, et al. [A comparative study of pragmatic language disorders and autism spectrum disorders using magnetoencephalography]. Rev Neurol. 2006;42 Suppl 2:S111-5. Spanish.

2. Allen DA, Rapin I. Language disorders in preschool children: predictors of outcome - a preliminary report. Brain Dev. 1980;2(1):73-80.

3. Bishop DV. The role of genes in the etiology of specific language impairment. J Commun Disord. 2002;35(4):311-28.

4. Botting N, Conti-Ramsden G. Autism, primary pragmatic difficulties, and specific language impairment: can we distinguish them using psycholinguistic markers? Dev Med Child Neurol. 2003;45(8):515-24.

5. Crespo-Eguílaz N, Narbona J. [Clinical profiles and transitions in the spectrum of specific language impairment in childhood]. Rev Neurol. 2003;36 Suppl 1:S29-35. Spanish.

6. Befi-Lopes DM. Avaliação, diagnóstico e aspectos terapêuticos nos Distúrbios Específicos de Linguagem. In: Ferreira LP, Befi-Lopes DM, Limongi SCO, organizadores. Tratado de fonoaudiologia. São Paulo: Roca; 2004. cap. 79. p. 987-1000.

7. Craig HK, Evans JL. Pragmatics and SLI: within-group variations in discourse behaviors. J Speech Hear Res. 1993;36(4):777-89.

8. Rollins PR, Pan BA, Conti-Ramsden G, Snow CE. Communicative skills in children with specific language impairments: a comparison with their language-matched siblings. J Commun Disord. 1994;27(2):189-206.

9. Bishop DV, Chan J, Adams C, Hartley J, Weir F. Conversational responsiveness in specific language impairment: evidence of disproportionate pragmatic difficulties in a subset of children. Dev Psychopathol. 2000;12(2):177-99.

10. Bishop DV, Norbury CF. Exploring the borderlands of autistic disorder and specific language impairment: a study using standardised diagnostic instruments. J Child Psychol Psychiatry. 2002;43(7):917-29.

11. Norbury CF, Bishop DV. Inferential processing and story recall in children with communication problems: a comparison of specific language impairment, pragmatic language impairment and highfunctioning autism. Int J Lang Commun Disord. 2002;37(3):227-51.

12. Crespo-Eguilaz N, Narbona J. [Subtypes of specific language impairment in Spanish-speaking children: a cluster analysis of linguistic features]. Rev Neurol. 2006;43 Suppl 1:S193-200. Spanish.

13. Bishop DV. Developmental cognitive genetics: how psychology can inform genetics and vice versa. Q J Exp Psychol (Colchester). 2006;59(7):1153-68.

14. Rocha LC, Befi-Lopes DM. Análise pragmática das respostas de crianças com e sem distúrbio específico de linguagem. Pró-Fono. 2006;18(3):229-39.

15. Liiva CA, Cleave PL. Roles of initiation and responsiveness in access and participation for children with specific language impairment. J Speech Lang Hear Res. 2005;48(4):868-83.

16. Conti-Ramsden G, Botting N. Social difficulties and victimization in children with SLI at 11 years of age. J Speech Lang Hear Res. 2004;47(1):145-61.

17. Stark RE, Tallal P. Selection of children with specific language deficits. J Speech Hear Disord. 1981;46(2):114-22.
18. Bishop DV. The underlying nature of specific language impairment. J Child Psychol Psychiatry. 1992;33(1):3-66.

19. Reed VA. Toddlers and preschoolers with specific language impairments. In: Reed VA. An introduction to children with language disorders. 2a ed. New York: Macmillan; 1994. p. 117-46.

20. Fernandes FDM. Pragmática. In: Andrade CRF, Befi-Lopes DM, Fernandes FDM, Wertzner HF. ABFW: Teste de linguagem infantil nas áreas de fonologia, vocabulário, fluência e pragmática. 1a ed. Carapicuíba: Pró-Fono; 2000. cap. 4. p. 77-89.

21. Fernandes FDM. Pragmática. In: Andrade CRF, Befi-Lopes DM, Fernandes FDM, Wertzner HF. ABFW: Teste de linguagem infantil nas áreas de fonologia, vocabulário, fluência e pragmática. 2a ed. Carapicuíba: Pró-Fono; 2004. cap. 4. p. 83-97.

22. Rodrigues A. Aspectos semânticos e pragmáticos nas alterações de desenvolvimento de linguagem. (Dissertação Mestrado). São Paulo: Faculdade de Filosofia, Letras e Ciências Humanas da Universidade de São Paulo; 2002.

23. Oller DK, Eilers RE, Neal AR, Schwartz HK. Precursors to speech in infancy: the prediction of speech and language disorders. J Commun Disord. 1999;32(4):223-45. Comment in: J Commun Disord. 1999;32(4):247-50.

24. Campbell TF, Dollaghan CA, Rockette HE, Paradise JL, Feldman HM, Shriberg LD, et al. Risk factors for speech delay of unknown origin in 3-year-old children. Child Dev. 2003;74(2):346-57.

25. Befi-Lopes DM, Palmieri TM. Análise dos processos fonológicos utilizados por crianças com alteração do desenvolvimento da linguagem. J Bras Fonoaudiol. 2000;1(4):48-58.

26. Tomasello M. Do young children have adult syntactic competence? Cognition. 2000;74(3):209-53. Comment in: Cognition. 2002;82(3):259-78.

27. Bedore LM, Leonard LB. Grammatical morphology deficits in Spanishspeaking children with specific language impairment. J Speech Lang Hear Res. 2001;44(4):905-24. Erratum in: J Speech Lang Hear Res. 2002;45(5): 1015.

28. Eadie PA, Fey ME, Douglas JM, Parsons CL. Profiles of grammatical morphology and sentence imitation in children with specific language impairment and Down syndrome. J Speech Lang Hear Res. 2002;45(4):720-32.

29. Windfuhr KL, Faragher B, Conti-Ramsden G. Lexical learning skills in young children with specific language impairment (SLI). Int J Lang Commun Disord. 2002;37(4):415-32.

30. Befi-Lopes DM, Gândara JP, Araújo K. Aquisição do sistema fonológico em crianças com alterações no desenvolvimento da linguagem. PróFono. 2003;15(1):19-30.

31. Grela B, Rashiti L, Soares M. Dative prepositions in children with specific language impairment. Appl Psycholinguist. 2004;25(4):467-80.

32. Wulfeck B, Bates E, Krupa-Kwiatkowski M, Saltzman D. Grammaticality sensitivity in children with early focal brain injury and children with specific language impairment. Brain Lang. 2004;88(2):215-28. 